\title{
HIGHLIGHTS
}

DEMENTIA

\section{Depression and dementia}

Two studies published recently in Neurology have further clarified the link between depression and dementiaidentified by previous research-by following large population-based samples over long follow-up periods.

In a 17 year follow-up, Jane Saczynski and colleagues found that depression at baseline was associated with a $>50 \%$ increase in dementia risk. Vonetta Dotson and colleagues showed that dementia risk increased with the number of episodes of elevated depressive symptoms (EDS) experienced by individuals over a period of nearly 25 years.

Several longitudinal studies have shown an association between depression and an increased risk of dementia in later life, but the findings have been inconsistent, perhaps due to variability in methodology and follow-up periods. Whether depression is a prodrome of dementia or a risk factor for this condition, or whether it simply shares some pathology with cognitive impairment, has remained unclear.

Saczynski and colleagues followed 949 participants (mean age at baseline 79 years) in the Framingham Heart Study for 17 years. $13.2 \%$ of the participants were depressed at baseline; that is, during the period 1990-1994. Over the follow-up period, $21.6 \%$ of the

\section{6 ...the results [from these studies] indicate that depression is a risk factor for dementia... 77}

participants with depression at baseline developed dementia, compared with only $16.6 \%$ of those who were depression free. Individuals who were depressed at baseline were more than 1.5 times as likely to develop dementia than those who were not depressed.

Dotson et al. monitored 1,239 participants (mean age at baseline 55.5 years) in the Baltimore Longitudinal Study of Aging over a follow-up period of up to 51 years (median 24.7 years). The individuals were assessed for dementia and depression at 1-2 year intervals. Participants who experienced one EDS had an $87 \%$ increased risk of developing dementia. "Each EDS was associated with a $14 \%$ increase in risk for all-cause dementia," says Dotson. She suggests that this dose-dependent relationship between depressive episodes and dementia risk is attributable to the underlying biological pathology, in that each EDS "results in neural insult to the brain and in turn increases the risk for dementia."
In view of the long follow-up times in both these studies, the results indicate that depression is a risk factor for dementia rather than a prodrome of the disease. "This finding highlights the importance of successfully treating depression in the elderly," says Dotson, as depression may be one of few modifiable risk factors for dementia.

Future research could focus on potential mechanisms for the link between depression and dementia. Saczynski plans to conduct such studies "perhaps via MRI," while Dotson and her team intend to focus on the underlying biological mechanisms, and investigate "structural and/or functional changes in the hippocampus that may be at play." The effects of interindividual differences on the relationship between depression and dementia also require further investigation.

Eleanor Beal

Original articles Dotson, V. M. et al. Recurrent depressive symptoms and the incidence of dementia and mild cognitive impairment. Neurology 75, 27-34 (2010) | Saczynski, J. S. et al. Depressive symptoms and risk of dementia: the Framingham Heart Study. Neurology 75 , 35-41 (2010) 